\title{
Emotion Detection from Text using Fuzzy Logic
}

\author{
Saqib Qamar \\ Dept. of Computer Science \\ Aligarh Muslim University
}

\author{
Parvez Ahmad \\ Dept. of Computer Science \\ Aligarh Muslim University
}

\begin{abstract}
In this paper we propose to use fuzzy logic in detecting emotional content from text. Fuzzy logic was developed to deal with concepts that do not have well-defined, sharp boundaries, which theoretically is ideal for emotion as no well-defined boundaries are defined for emotion categories (e.g., happiness, sadness, surprise, fear, disgust, and anger).The transition from one physiological state of emotion to another is also gradual and could easily be modeled by fuzzy logic approach.
\end{abstract}

\section{Keywords}

Fuzzy logic, emotion, text.

\section{INTRODUCTION}

The procedure of how to extract emotions from text is given below in figure 1.

Expression of emotional feeling between people forms an interactive web. This complex interactional emotion is fundamental to human lives and decision-making. Understanding this phenomenon is a hot topic for research.

Previous works have concluded that emotions can be detected in various ways- in speech, in facial expressions, and in text. As text is still dominating online communications, we believe that emotion detection in textual messages is particularly important. In this paper we are using fuzzy logic for emotion detection.We want to introduce about fuzzy set.

\subsection{Fuzzy Set Theory}

The essential to extract knowledge from domain experts or from training data obtained in the real world, known as knowledge acquisition, is a task chief to applications of knowledge engineering. However, the imprecision intrinsic to human knowledge and to the real world makes this task quite difficult to accomplish using a conventional scientific model, as such a model attempts to precisely represent all the characteristics of the expert system we are building and absence of flexibility. Fuzzy set theory, introduced by Zadeh [2], should provide the needed tools needed to deal with the vagueness of our knowledge, and allows us to represent the parameters of a system using unclear linguistic terms rather than exact mathematical values. However, we are left with the task of building the affiliation functions of these parameters and the Fuzzy rules of the system. This is an important block in the construction of fuzzy expert systems, and if done manually, would require expensive resources from domain experts and knowledge engineers [4]. Fortunately, for most real-world applications, numerical data can be easily obtained from tools or from the environment. Several researchers have come up with ways to use this data to allow the system to construct its own fuzzy rules, using various types of learning algorithms. For example, Wang and Mendel [5] used a tablelookup technique to generate their rules. A more prevalent approach is to use neural networks [7] [9], modular neural networks [8] or hybrid systems [6] to extract knowledge from the training examples and build the rules. Hoang and Lee [10] proposed a simpler learning system, based only on fuzzy clustering and decision tables, that is able to defining the membership functions and the fuzzy rules without relying on complex computational schemes like neural networks. However, the size of their decision table grows with the number of input variables, and the system develops overly complicated in accurate real-world applications, which may involve a very high number of variables. Wu and Chen [3] have been proposed another simple learning algorithm. Their technique divides the training data into clusters the using the a-cuts of fuzzy equivalence relations and of fuzzy sets, and then derives the membership function of each of these clusters. This learning algorithm generates on average half the number of rules the one by Hoang and Lee generates, but despite that easiness it earns slightly more accurate results that Hoang and Lee's system.

\subsection{Significance of Emotions}

Some of the practical applications based on emotions are:

\subsubsection{Applications in Business and Banking Sectors}

Emotions and opinions have great impact on customers to make their choices regarding online shopping, choosing events, products and entities. These opinions also help the banks to design plans and schemes for insurance sector.

\subsubsection{Applications in Politics and Exit Polls}

As it is well known, opinions and emotions matter a great deal in politics, proposed work has high utility on understanding what voters are thinking, what public figures support or not support the government and exit polls forecasting election results.

\subsubsection{Applications in Detecting Email Spams}

Application of the proposed work has high utility in detecting email spams. 


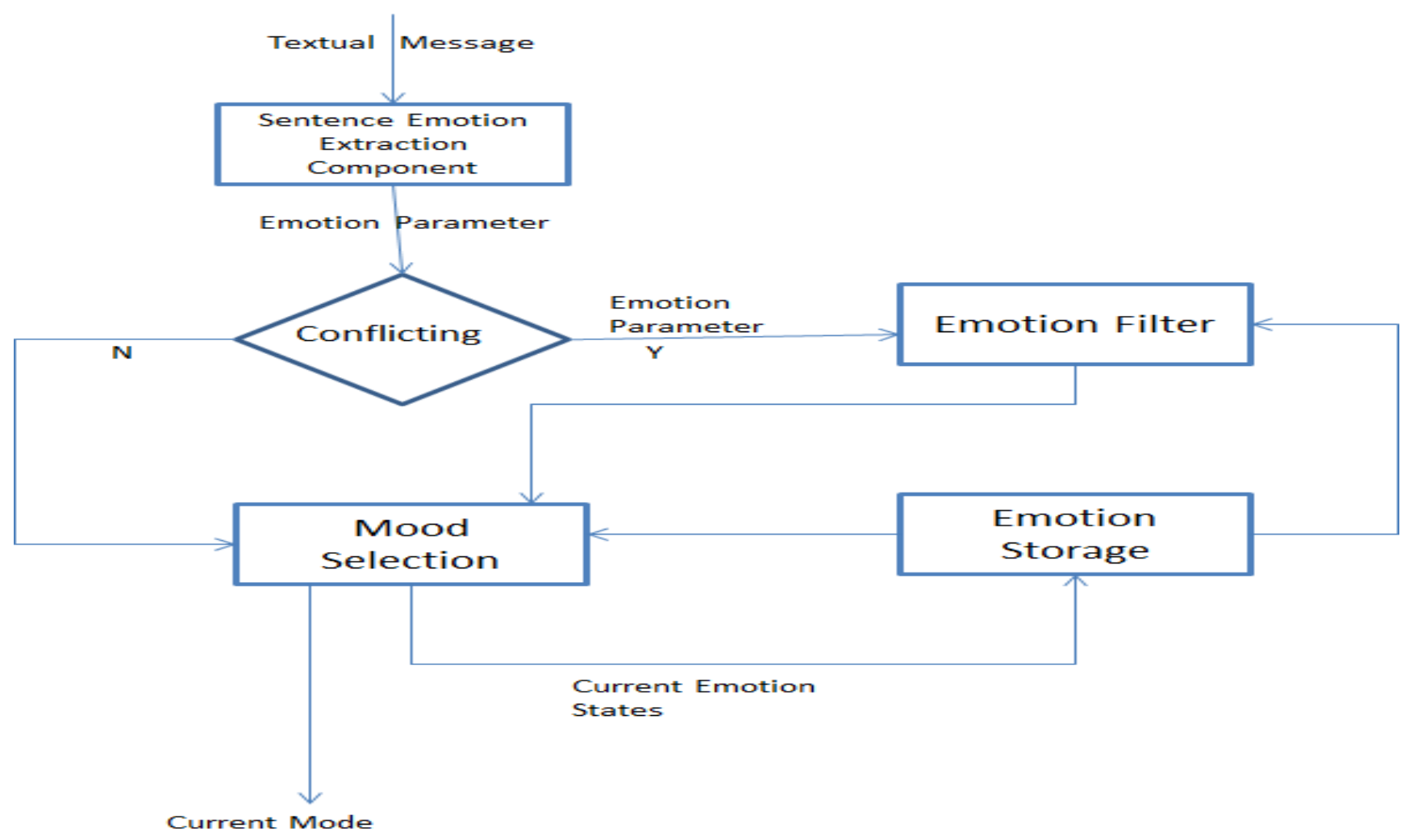

Figure 1. Emotion Extraction Engine overview

\section{PROPOSED WORK}

To build an intelligent emotion extraction engine that can recognize complex human emotions from textual contents using fuzzy logic.

\section{PROPOSED SOLUTION}

The emotion extraction engine is a generic prototype based on keyword tagging and real-world knowledge. Figure 1 [11] depicts an overview of the architecture of the emotionextraction engine. The engine classifies emotions into the following categories: happiness, sadness, surprise, fear, disgust, and anger.

The user's mood is defined as the feelings perceived from a user's series of input in the emotion extraction engine. The current emotion of a user is based totally on the information assessed within a single sentence. A user's mood may not be consistent with the current emotion of the user. For example, a user may present a sad feeling in one sentence, but previously the user was talking about happy and interesting things. The sad feeling presented may not be a significant emotion and overall the user's mood may be still happy. To calculate the mood of a user, previous emotions and current emotions need to be analyzed together.

\subsection{Conflicting Emotion Detection}

An emotion is a common phenomenon in daily life, it is not uncommon for a user to type in a sentence, such as, "I am very happy that I got a job, but I am sad that I have to work overtime" that contains mixed emotions in an online chatting environment. When sentences contain conflicting emotions refereeing which emotion represents the overall emotional feeling is not only based on the current sentence, but also on the mood. For example, in the emotion extraction engine, the mood happy indicates that the previous messages an individual typed contain overpoweringly more happy feelings than others. When the user types a sentence containing both happy and sad emotions, the perceived current mood of the user may still be happy instead of happy and sad. The reason is that the individual was in a primarily happy mood, and the presented sad emotion may not be significant enough to change the mood from happy to sad.

The negative emotion category and positive emotion category are presented to handle different type of emotions. Positive emotions include happiness and surprise, while negative emotions are sadness, fear, anger, and disgust. A sentence is called conflicting- emotion sentence only if the sentence contains both negative and positive emotions.

If the sentence has conflicting emotions then the emotion filters the dominant emotion.

\subsection{Mood Selection Component}

The input of the mood-selection component is the emotion parameter from the sentence emotion extraction component and the previously stored emotions in the emotion-storage component. The purpose of the mood selection component is to conclude the current mood.

To achieve this, the first step of the mood selection component is to convert the emotion parameters into the current emotions by filtering the tense information. For example, the emotion parameter [happiness][middle intensity][present sentence] is converted to the current emotion [happiness][middle intensity]. The current emotion is sent to the storage component as well. The previous emotions 
of the user are stored in the storage component. The format is [emotion category] [intensity]. To covert the emotion data into the format acceptable for a fuzzy system, the following fuzzy-data calculations are carried out.

\subsection{Fuzzy Data Calculation}

An array $\mathrm{E}$ is assigned to contain the accumulative intensity values of the seven emotion categories. The array elements 0 to 6 in turn represent the accumulative intensity of the emotions happiness, surprise, anger, disgust, sadness, excited and fear. The value of each element in array $\mathrm{E}$ is calculated by adding the six previous intensities of a specific emotion category with the current intensity of that emotion. Equation $1[1]$ applied to calculate the accumulative intensity is shown as follows.

$E[x]=\sum_{i=-n}^{0} \alpha_{i} I_{i}(x)$, where $x=0,1,2,3,4,5,6$

The values of array $\mathrm{E}$ depend on the relative intensity over the last $\mathrm{n}$ time periods; $\mathrm{n}$ is chosen to be 6 as it is assumed that in a chatting environment users only remember the most recent dialogs. $\mathrm{Ii}(\mathrm{x})$ is the intensity of emotion category $\mathrm{x}$ at discrete time $\mathrm{i}$, and the value of $\operatorname{Ii}(\mathrm{x})$ varies from 0 to 3 , which represents the lowest intensity to the highest intensity. When $\mathrm{i}$ is 0 , $\operatorname{Ii}(x)$ contains the intensity values of the current emotions.

In the emotion extraction engine, the negative and positive emotions are assumed to decay at the same rate and the influence period is chosen to be five sentences. Figure 2 illustrates the assumption. In Figure 2[13], Value represents the value of the perceived influence of emotion and $t$ represents time.

EMO represents the emotion that occurred at the distinct time point (e.g., the time when a chat message is input). In this figure, the value of EMO at different time points is the same, which means that a user typed six emotional sentences of the same intensity. Zero represents the current time. However, at time point 0 , the perceived influence of EMO that occurred at time -5 is 0 , which means the influence of the emotion input at time -5 has disappeared. The EMO that occurred at time -1 is the least decayed and has the strongest influence at the current time (time 0). In the fuzzy emotion-analysis component, the emotion decay is represented by the weight parameter, and it is calculated using Equation 2[1]

$$
\begin{aligned}
& \alpha_{i}=\alpha_{i+1}-0.1, \quad \text { where } i=-5,-4,-3,-2 \\
& \alpha_{-1}=0.5 \\
& \alpha_{0}=1
\end{aligned}
$$

\subsection{Fuzzy Membership Functions}

The following fuzzy logic rules and fuzzy membership functions are applied to assist the mood calculation. Two fuzzy membership functions high and low (Equations 3 and 4) [1] are defined to guide the analysis of fuzzy functions.

$$
\begin{aligned}
& \operatorname{high}(x)=\frac{E[x]}{\sum_{i=0}^{5} E[i]} \\
& \operatorname{low}(x)=1-\frac{E[x]}{\sum_{i=0}^{5} E[i]}
\end{aligned}
$$

\subsection{Fuzzy Rules}

Fuzzy rules are created based on the high and low membership functions. The rule base includes the following.

Rule 1:

IF the emotion with largest intensity is "high" AND the other emotions are "low"

THEN the current mood is that emotion

Rule 2:

IF the positive emotions are "high AND the negative emotions are "low"

THEN the current mood is the highest positive emotion and the intensity is decreased by 0.1

Rule 3:

IF the negative emotions are "high" AND positive emotions are "low"

THEN the current mood is the highest negative emotion and the intensity is decreased by 0.1

\subsection{Mood Selection}

When a dialog starts, there are no cues as to what mood a user is in. However, it is reasonable to assume that the mood of a new user is neutral. As the emotion extraction engine acquires more data (e.g., the user starts chatting), the above fuzzy rules can be applied and the mood of the user can be calculated. The center of gravity (COG) point is an important measurement factor in determining the dominant rule. In this implementation, the COG point is calculated as the average of the rules' outputs (Equation 5) [1]

$$
C O G=\frac{\sum_{1}^{3} \operatorname{rule}(x)}{3}
$$

\section{CONCLUSIONS AND FUTURE WORK}

Emotion detections have a promising future. Major methodology such as fuzzy logic towards Emotion Extraction from text has been discussed in this paper. Although not enough time has passed to have established standards in the field, there is some consistency between the approaches, and the algorithms are continuing to increase in accuracy. Although various rules and algorithms applied in the emotion extraction engine, the engine still cannot handle the difficulty of English language and the boundaries of the emotion extraction engine are summarized in this section. However, the emotion extraction engine still can be applied within the Internet communication context and achieve considerable performance. As the technology develops, some of the current limitations may be solved in the near future. 


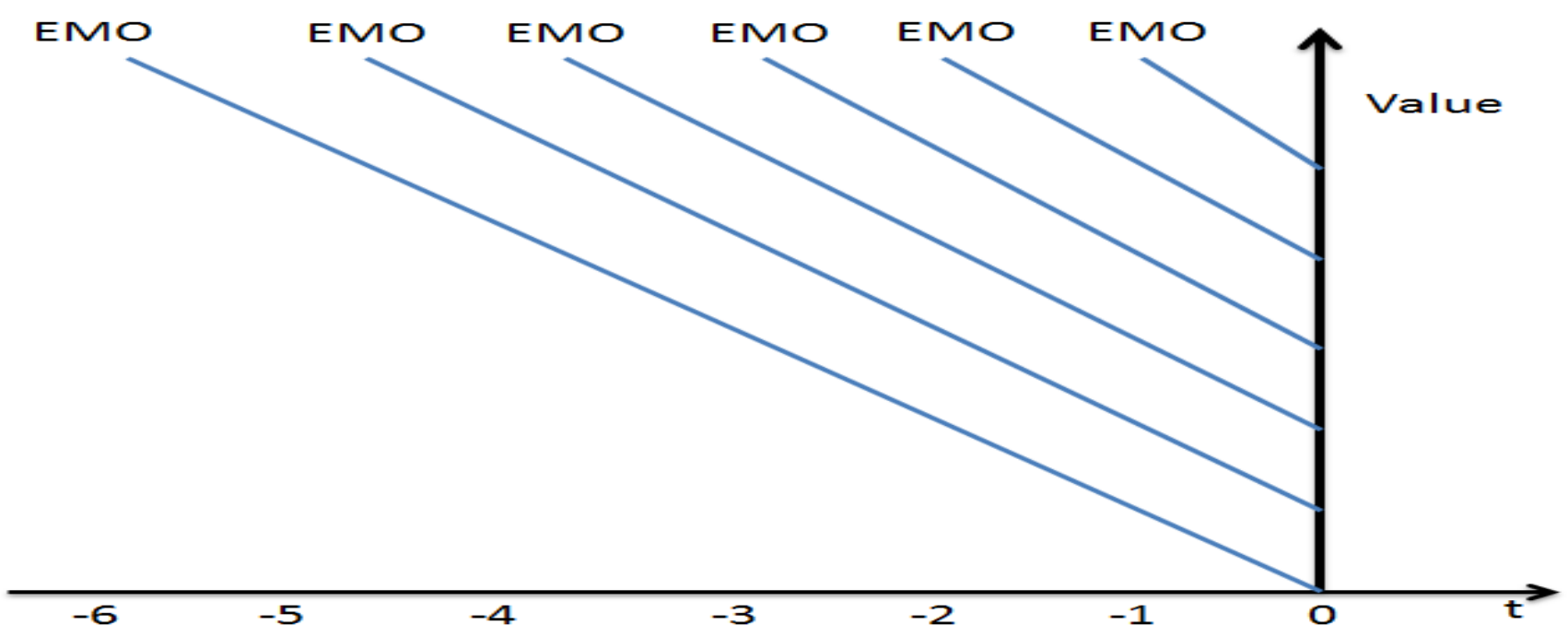

Figure 2. The decay of emotion over time

\section{REFERENCES}

[1] Encyclopedia of human-interaction by "CLAUDE GHAOUT".

[2] L. A. Zadeh, "Fuzzy sets", Information and Control, Vol. 8, 1965, pp. 338-353.

[3] T-P Wu, S-M Chen, "A new method for constructing membership functions and fuzzy rules from training examples", IEEE Transactions on Systems, Man and Cybernetics, Part B, Volume 29, Issue 1, February 1999, pp. 25-40.

[4] J. C. Cano, P. A. Nava, "A fuzzy method for automatic generation of membership function using fuzzy relations from training examples", Proceedings of the Annual Meeting of the North American Fuzzy Information Processing Society, 27-29 June 2002, pp. 158-162.

[5] L. X. Wang, J. M. Mendel, "Generating fuzzy rules by learning from examples, IEEE Transactions on Systems, Man and Cybernetics, Vol. 22, Issue 6, 1992, pp. 14141427.

[6] N. K. Kasabov, "Learning fuzzy rules and approximate reasoning in fuzzy neural networks and hybrid systems", Fuzzy Sets and Systems, Vol. 82, no. 2, 1996, pp. 135149.

[7] J. J. Shann, H. C. Fu, "A fuzzy neural network for rule acquiring on fuzzy control systems", Fuzzy Sets and Systems, Vol. 71, No. 3, 1995, pp. 345- 357.

[8] R. Langari, L. Wang, "Fuzzy models, modular networks, and hybrid learning", Fuzzy Sets and Systems, Vol. 79, No. 2, pp. 141-150, 1996.
[9] C. T. Lin, C. S. G. Lee, "Neural-network-based fuzzy logic control and decision system", IEEE Transactions on Computers, Vol. 40, Issue 12, 1991, pp. 1320- 1336.

[10] T. P. Hong, C. Y. Lee, "Induction of fuzzy rules and membership functions from training examples", Fuzzy Sets and Systems, Vol. 84, No. 1, 1996, pp. 33-47.

[11] Mohamed Yassine, Hazem Hajj (2010), “A Framework for Emotion Mining from Text in Online Social Networks", IEEE International Conference on Data Mining Workshops, Sydney, NSW, IEEE publications, Dec 2010, pp. 1136-1143.

[12] Esuli Baccianella Stefano, Esuli Andrea and Sebas-tiani Fabrizio, (2010), "SentiWordNet 3.0: An Enhanced Lexical Re-source for Sentiment Analysis and Opinion Mining". In Procedings of the 7th Conference on Language Resources and Evaluation, pp. 2200-2204.

[13] Turney Mohammad, S. and Turney, P.D. (2010), "Emotions Evoked by Common Words and Phrases: Using Mechanical Turk to Create an Emotion Lexicon". Proceedings of the NAACL-HLT 2010 Workshop on Computational Approaches to Analysis and Generation of Emotion in Text, Los Angeles, ACM Publications,June 2010, pp. 26-34.

[14] Isa Maks, Piek Vossen, (2011), “A Verb Lexicon Model for Deep Sentiment Analysis and Opinion Mining Applications", Proceedings of the 2nd Workshop on Computational Approaches to Subjectivity and Sentiment Analysis, ACL-HLT 2011, Portland, Oregon, USA, ACM publications, June 2011, pp. 10-18. 